\section{Acknowledgements}

This study was supported by key program of Science and Technology Department Foundation of Fujian Province (Grant No 2007N0050). We also thank Dr Mark Roberts for constructive comments on this manuscript.

\section{References}

Adams S, Mapstone BD, Russ GR, Davies CR (2000) Geographic variation in the sex ratio, sex-specific size and age structure of Plectropomus leopardus (Serranidae) between reefs open and closed to fishing on the Great Barrier Reef. Canadian Journal of Fisheries and Aquatic Sciences, 57, 1448-1458.

Armsworth PR (2001) Effects of fishing on a protogynous hermaphrodite. Canadian Journal of Fisheries and Aquatic Sciences, $58,568-578$.

Armsworth PR (2002) Recruitment limitation, population regulation and larval connectivity in reef fish metapopulations. Ecology, 83, 1092-1104.

Frisch A, Anderson T (2005) Physiological stress responses of two species of coral trout (Plectropomus leopardus and Plectropomus maculatus). Comparative Biochemistry and Physiology A-Molecular \& Integrative Physiology, 140, 317-327.

Heemstra PC, Randall JE (1993) FAO Fisheries Synopsis. No. 125, vol. 16, pp. 292-293. FAO, Rome. van Herwerden L, Choat JH, Dudgeon CL et al. (2006) Contrasting patterns of genetic structure in two species of the coral trout Plectropomus (Serranidae) from east and west Australia: Introgressive hybridisation or ancestral polymorphisms. Molecular Phylogenetics and Evolution, 41, 420-435.

Munday PL, Jones GP, Pratchett MS, Williams AJ (2008) Climate change and the future for coral reef fishes. Fish and Fisheries, 9, 261-285.

Nei M (1973) Analysis of gene diversity in subdivided populations. Proceedings of the National Academy of Sciences of the United States of America, 70, 3321-3323.

van Oosterhout C, Hutchinson WF, Wills DPM, Shipley P (2004) MICRO-CHECKER: software for identifying and correcting genotyping errors in microsatellite data. Molecular Ecology Notes, 4, 535-538.

Sambrook J, Fritsch EF, Maniatis T (1989) Molecular Cloning: A Laboratory Manual, 2nd edn. Cold Spring Harbor Laboratory Press, Cold Spring Harbor, NY.

Yeh FC, Yang RC, Boyle T (1999) PopGene (version 1.32). Microsoft Window-base Software for Population Genetic Analysis: A Quick User's Guide. University of Alberta, Center for International Forestry Research, Alberta, Canada.

doi: 10.1111/j.1755-0998.2009.02690.x

(C) 2009 Blackwell Publishing Ltd

\title{
Microsatellite markers associated with genes expressed in developing wings of Bicyclus anynana butterflies
}

\author{
PATRÍCIA BELDADE, ${ }^{*}+$ ARJÈN E. VAN’T HOF, ł MARIA ADELINA JERÓNIMO† and ANTHONY D. \\ LONG§ \\ *Institute of Biology Leiden University, PO Box 9516, 2300 RA, Leiden, The Netherlands, †Instituto Gulbenkian de Ciência, \\ Apartado 14, P-2781-901 Oeiras, Portugal, ¥School of Biological Sciences, Biosciences Building, Crown Street, University of \\ Liverpool, Liverpool L69 7ZB, UK, §Department of Ecology and Evolutionary Biology, University of California at Irvine, 5210 \\ McGaugh Hall, Irvine, 92697-2525 CA, USA
}

\begin{abstract}
Deriving useful microsatellite markers in lepidopterans has been challenging when relying on scans of genomic DNA libraries, presumably due to repetitiveness in their genomes. We assayed 96 of 320 microsatellites identified in silico from a collection of Bicyclus anynana ESTs, in 11 independent individuals from a laboratory population. From the 68 successful assays, we identified 40 polymorphic markers including 22 with BLAST-based annotation. Nine of 12 selected polymorphic markers tested in a panel of 24 wild-caught individuals converted to successful assays and were all polymorphic. We discuss how microsatellite discovery in ESTs is an efficient strategy with important attendant advantages.
\end{abstract}

Keywords: Bicyclus anynana, EST, genetic markers, Lepidoptera, microsatellite, polymorphism

Received 20 June 2008; revision accepted 26 February 2009

Correspondence: Patrícia Beldade, Fax: +31 71 5274900;

E-mail: p.beldade@biology.leidenuniv.nl
Microsatellites are widely used as neutral genetic markers for a variety of purposes; including analysis of relatedness, population structure, demographic and selective 
processes, and genetic mapping (Ellegren 2004; Schlötterer 2004). Moreover, several studies have suggested that microsatellites can also play important roles in generating variation (Rockman \& Wray 2002; Fondon \& Garner 2004; Kashi \& King 2006). Microsatellites have been explored in a variety of organisms, but have proven difficult to obtain for lepidopterans (butterflies and moths) using traditional methods that rely on genomic DNA libraries. This is potentially because lepidopteran microsatellites often appear within multi-copy genomic regions (Zhang 2004, Van't Hof et al. 2007), which results in PCR failure.

Lepidopterans have long been favourite targets of biological research (also because of their economic importance), and have some unusual genetic properties (such as heterogametic females and holocentric chromosomes). More recently, the derived and diverse colour patterns on butterfly wings have been the focus of efforts to develop genomic resources to link genotypes, to development, to phenotypes, to fitness (Beldade et al. 2008). Linkage maps are now available for a few species (Jiggins et al. 2005; Kapan et al. 2006; Van't Hof et al. 2008, Wang \& Porter 2004), but are made mostly of anonymous markers whose value for across-species comparisons (e.g. Yasukochi et al. 2006; Pringle et al. 2007; Beldade et al. 2009) is limited. Having markers associated with expressed genes has advantages for downstream use of the maps and should improve marker conversion success. Growing EST collections for butterflies (Papanicolaou et al. 2008) represents a potential valuable source for microsatellites in this group. Here, we analyse microsatellites found in an EST collection from Bicyclus anynana butterflies, an emerging model for the evolutionarydevelopmental analysis of adaptive phenotypes (Beldade \& Brakefield 2002).

Our B. anynana EST collection was derived from developing wings of a large number of outbred individuals to combine gene discovery with DNA sequence polymorphism identification (Beldade et al. 2006). A custom PERL script designed to search for microsatellite repeats detected 320 di-, tri-, tetra- and penta-nucleotide repeat microsatellites (perfect repeats with repeat number greater than 5 for dinucleotide microsatellites and greater than 3 for the remainder) in 4251 assembled unigenes (Beldade et al. 2006). Ten of the 73 microsatellites in nonsingleton unigenes were polymorphic in our EST collection with up to seven alleles.

We selected 96 of the 320 EST-based microsatellites to test in a panel of 11 individuals (five females and six males) from a laboratory outbred stock (Brakefield et al. 2009). To maximize the likelihood of finding polymorphisms, we identified 151 microsatellites that were either polymorphic in the EST collection (Beldade et al. 2006) or that had relatively high repeat number (dinucleotides with greater than 5 repeats, trinucleotides with greater than 4 repeats, and tetra- and pentanucleotides with greater than 3 repeats). Primer3 software (Rozen \& Skaletsky 2000; default settings unless noted) was used to design primers (optimal size $=22$, and range $=20-25$ ) to amplify a fragment of $100-150 \mathrm{bp}$ around the microsatellite, avoiding priming low Phred and potential polymorphic bases (Beldade et al. 2006). Of the 151 original target microsatellites, this process led to automated design of 94 primer pairs (design failure was typically associated to microsatellites near the end of the available sequence). To increase the target group to 96, we manually selected two extra microsatellites from the 320 . These were trinucleotides with 4 repeats in two unigenes annotated as Ribosomal Proteins (contigs 702 and 835 in Table S1). In total, primer pairs were designed to amplify 96 microsatellite loci in 94 unigenes, of which 60 were annotated based on sequence similarity with public databases (Beldade et al. 2006).

A 5' M13-tag (TGTAAAACGACGGCCAGT) was added to each of the 96 forward primers to enable the use of a single fluorescently labelled primer (M13-FAM, cf. Schuelke 2000). 0.5 pmol forward and 2 pmol reverse

Table 1 Microsatellite markers in Bicyclus anynana derived from genomic and cDNA libraries

Mono

Source Found Assayed PCR Called morphic Polymorphic

\begin{tabular}{|c|c|c|c|c|c|}
\hline cDNA 320 & 96 & 76 & 68 & 28 & $\begin{array}{l}40 \text { [18] } \\
2 \text { alleles }=18[6] \\
3 \text { alleles }=15[7] \\
4 \text { alleles }=5[3] \\
>5 \text { alleles }=2[2\end{array}$ \\
\hline gDNA 252 & 80 & 51 & 41 & 13 & $\begin{array}{l}28 \\
2 \text { alleles }=6 \\
3 \text { alleles }=6 \\
4 \text { alleles }=10 \\
>5 \text { alleles }=6\end{array}$ \\
\hline
\end{tabular}

Microsatellites identified in cDNA (Beldade et al. 2006) and gDNA (Van't Hof et al. 2005) libraries were typed in 11 and 28 outbred individuals, respectively. Counts are given for: (i) the total number of microsatellites identified (Found) following specific criteria (see Van't Hof et al. 2005; and Beldade et al. 2006), (ii) the subgroup for which primer pairs were designed and tested in genomic DNA (Assayed), (iii) primer pairs for which the PCR was successful (PCR), i.e. excluding those for which the microsatellite had either no bands on any of the test individuals or had bands for fewer than 5 of the 11 test individuals, (iv) microsatellites that we were actually able to type (Called), i.e. excluding failed PCR and inconclusive and (v) the number of Monomorphic and Polymorphic markers among the latter. Numbers in square brackets correspond to insertion-deletion polymorphisms or polymorphic microsatellites with imperfect repeats (see text). 
primer, and 2 pmol M13-FAM (IDT) were used in a $12 \mu \mathrm{L}$ PCR reaction with ca. $10 \mathrm{ng}$ of genomic DNA (QIAGEN DNeasy), 0.3 U Taq polymerase (MasterTaq,
Eppendorf) with buffer provided and enhancer additive ( $0.5 \times$ final concentration), and $10 \mathrm{nmol}$ dNTPs. Denaturing at $95^{\circ} \mathrm{C}$ during $3 \mathrm{~min}$ was followed by 10 cycles of

Table 2 EST-based microsatellite markers polymorphic in a laboratory population

\begin{tabular}{|c|c|c|c|c|c|c|c|c|c|c|}
\hline ID & BLAST & Forward primer & Reverse primer & $\begin{array}{l}\text { rep } \\
\text { seq }\end{array}$ & $\begin{array}{c}\text { rep } \\
\text { nr }\end{array}$ & $\begin{array}{l}\text { poly } \\
\text { EST }\end{array}$ & alleles & $\begin{array}{l}\text { size } \\
\text { range }\end{array}$ & indiv & HW \\
\hline$\underline{120}$ & 1 & GGTAAAACAATTCGATTATCAACA & TTGGACATCAATGATACTGAAGTTA & ATT & 5 & 1 & 2 & 116-142 & 11 & 1 \\
\hline 213 & 0 & ACTTGAACCTAAAACACCAGCC & AGAAGTACGAACAACGAACACG & TGACT & 5 & 0 & 3 & 104-119 & 10 & 0.543 \\
\hline 248 & 0 & TGACATAGAGAGCTCCTGGGAT & CACAGAGACATGAAGATGAGTGC & $\mathrm{AC}$ & 6 & 1 & 2 & $118-120$ & 11 & 0.542 \\
\hline 349 & 1 & AGATTTTGGGCGGAATCTACTT & AAAAGTACGACCCAACACAAGC & ACTA & 4 & 0 & 2 & $146-148$ & 8 & 0.016 \\
\hline 438 & 1 & GTTGTCGTTTTGTTGGGTGGTA & GTCAGAGACGCACCGATCTAAC & GAGT & 8 & 1 & 2 & 100-104 & 8 & 0.200 \\
\hline 455 & 1 & AAATCGGACCAGTAGTTCCAGA & GAAATACGGAAAGAGGTTGACG & CAAA & 5 & 1 & 3 & 123-131 & 11 & 0.143 \\
\hline 572 & 0 & TTGTGTTGCAATATGGTTAGGC & CAGCTGCAGTTTGTTACTACCAC & AATA & 4 & 0 & 2 & $134-138$ & 10 & 1 \\
\hline 575 & 1 & ACGTCCATCGCCTGTTCATTAT & TGCAAAATATCGTTTTATTTCAGA & ATG & 5 & 0 & 3 & 142-146 & 10 & 1 \\
\hline 698 & 1 & GACATTATTTCACTTCAACAACAGG & AAATCCAATTCAAGGAATAACAAC & TA & 6 & 0 & 2 & $127-129$ & 11 & 0.108 \\
\hline 780 & 1 & CACGTAAAATGTTGCGTTTTATT & GAGAAGATGTCCAGATTCCTCG & AAT & 5 & 0 & 3 & $147-156$ & 11 & 1 \\
\hline$\underline{818}$ & 1 & TCGCATATTAAGTCTTCAAGCA & TAGACCCACTGAAATGAAAGGG & TTC & 4 & 1 & 5 & $142-156$ & 11 & 0.005 \\
\hline 820 & 1 & GCGTCGAGTGTGCTACAGTTAT & GCCTTACGAACAACACGAGTTTA & TTAT & 4 & 0 & 4 & $117-128$ & 10 & 1 \\
\hline 902 & 1 & TAACGGAGTTCGTTTATTTCGG & CAACACCGATTGTCCTCTACAA & AT & 6 & 0 & 2 & $118-120$ & 11 & - \\
\hline 1006 & 1 & TCAAATCGGACCAGTAGTTCCT & CCATTTTCAAGTTTGTCACTATTCT & CAAA & 5 & 0 & 2 & $127-131$ & 6 & - \\
\hline 1369 & 0 & TTGTTTTTGTTTTGTGCAATAAAGT & AGGTTGTCTCATGTAAGCGTTG & ATAA & 6 & 0 & 2 & 127-135 & 11 & 0.232 \\
\hline 1373 & 1 & CACAGGCCAGTATTATGTAAGAGA & TTCATCATAGTGGGTACAACAAAAA & AC & 7 & 0 & 5 & 153-160 & 11 & 1 \\
\hline 1710 & 1 & GCAATTTGCATTTTCAATACCA & CGTTGACTAACTCTTAGCTTTGACA & CA & 6 & 0 & 3 & $117-123$ & 11 & 0.050 \\
\hline 1808 & 0 & AAAATCCAACCCAAACAACAAC & TGTTGGTGATCACACTGAACAA & ATT & 7 & 0 & 3 & $143-149$ & 7 & 0.076 \\
\hline 1876 & 1 & TGAATCGCTTCATTCTTTCATA & AATTGAGGAAAATAACGCAGGT & GTAG & 6 & 0 & 4 & $122-136$ & 9 & 0.525 \\
\hline 1899 & 0 & ATTTGAATCGCGCATTTATTTT & TATGCAAATTTTATTTCGGGCT & ATA & 6 & 0 & 2 & $156-158$ & 10 & 1 \\
\hline 2339 & 0 & TGTTGAAAACAAAATTAACAAAACA & TCTGGATCCTGTAACAAACGTG & AT & 6 & 0 & 4 & 131-137 & 11 & 0.008 \\
\hline 2484 & 0 & GGCGCGTACCAACTACATACTTA & TTGAAACAAAATAAACATTTTGGAA & ATT & 5 & 0 & 2 & $123-130$ & 10 & 1 \\
\hline 2555 & 0 & TTAAACTTCCTGGTCCACAGGT & TAAGTTTGACCCACCTTTTGCT & TTAA & 4 & 0 & 2 & 140-144 & 11 & - \\
\hline 2562 & 0 & GTAATGTCTTCCCGGGTATTGA & AAAATATTGCGAGTAGGTTGCC & TAAA & 7 & 0 & 3 & 135-143 & 8 & 0.513 \\
\hline 2577 & 1 & TTTTCTTCATTATGACCCCCTG & GATTTGCCAGAAGAACCAGAAA & TTC & 5 & 0 & 2 & $152-163$ & 11 & 0.255 \\
\hline 2636 & 0 & AGACGGGAGTGAGAAAAGAGC & GGCAGAACGAATATTTGAGTCC & TTCA & 4 & 0 & 3 & 115-201 & 10 & 1 \\
\hline 2669 & 0 & TGTTGTTTAATTGGAAGTTTTCAGA & CGTGGGAAATTGGGAATATAGA & ACA & 5 & 0 & 3 & 138-147 & 9 & 0.525 \\
\hline 2904 & 1 & AAATTAGCGTATCGATCAGTACAA & GAACTCAGTAACAATATGCTTGGAA & TAAT & 4 & 0 & 2 & $136-140$ & 11 & 1 \\
\hline 3167 & 0 & TGGAATGTTGTATTCTTGCAAAT & AGGCGTTGCTTAGATATTCTGG & ATT & 5 & 0 & 2 & 156-159 & 11 & 0.279 \\
\hline 3410 & 0 & CCCATAATATTGGCTTAATGGT & GAGCGGTCCACTTAGTGAAGTT & TTTA & 4 & 0 & 2 & 139-147 & 11 & 1 \\
\hline 3457 & 1 & CACACGGCTTTTTGTGTATTTT & CGATTTTGCTAAGCATGTTAGTTC & TTTA & 5 & 0 & 4 & $155-160$ & 11 & 0.509 \\
\hline 3655 & 0 & CCTAGTCATCATCCCGATTCTT & CATAGTCGGATAGTTCATCAATACA & TTA & 5 & 0 & 2 & $127-130$ & 10 & 1 \\
\hline 3752 & 1 & ACTTTCGAAACGTCCGGTAATA & CGATGACTAGATCACGGAAACA & ATAA & 4 & 0 & 3 & 164-174 & 9 & 0.021 \\
\hline 3833 & 1 & AACCAGTATTCTTGCCACCATT & TGCCCTACAGTAAACATTCATCA & TCA & 5 & 0 & 3 & 149-161 & 10 & 0.011 \\
\hline 4122 & 1 & ACCGCCTTTGCACATACTTATT & TCAATGCAAACAAACAAACAAA & AAAT & 4 & 0 & 3 & $146-154$ & 10 & 0.173 \\
\hline 4122 & 1 & TGTTATTGTTTTTGTTTTGTGCAAT & GCTTCCCAACAGTGGATTTTTA & GTTT & 4 & 0 & 4 & 154-166 & 9 & 0.020 \\
\hline 4190 & 0 & ATTATGCACAGCACTCTGTCGT & TAGGATTGGAGAACGGAACGTA & CA & 6 & 0 & 3 & 133-137 & 10 & 1 \\
\hline 4316 & 0 & TTCGCGAATATAACGTGAAAAA & TGTGGATATTTCACAGCAAAAA & GT & 7 & 0 & 3 & 155-164 & 10 & 0.001 \\
\hline 4431 & 1 & TCCTACACTATGCGGTTCACTG & ACATCGACTACAACAACAACGC & TGT & 5 & 0 & 2 & $101-122$ & 11 & 1 \\
\hline 4442 & 0 & TGGGTTCGAAATAAACGCAT & CGGTACATTTCATCCTGTCAGA & TTTC & 4 & 0 & 3 & $145-155$ & 11 & 0.079 \\
\hline
\end{tabular}

Data on the microsatellite markers identified in silico in a B. anynana EST collection (Beldade et al. 2006): ID is the contig number with underlining marking 'indels' and italics for markers tested also in a wild-caught panel (NCBI ESTdb accession numbers and contig consensus sequences are provided in Supplementary Table S1); BLAST is a binary code with 1 for unigenes annotated based on BLAST analysis against publicly available sequences, and 0 to those with no such annotation (details in Supplementary Table S1); rep seq is the microsatellite motif; rep $n r$ is the number of repeats in the microsatellite in ESTs; and poly EST is a 0/1 code with 1 for the microsatellites found to be polymorphic in the EST collection. The remaining columns refer to the analysis in this paper: sequences of the Forward and reverse primers designed to type each microsatellite; alleles is the number of alleles found in the test panel; size range is the range for the length ( $\mathrm{bp}$ ) of the amplicons detected in the laboratory panel; indiv is the number of individuals from the panel genotyped for each microsatellite marker; $H W$ is the p-value for the test for deviations from Hardy-Weinberg equilibrium [calculated with GENEPOP (Rousset 2008) based on the genotype data in Supplementary Table S1] with bold indicating significant deviations and - for alleles that did not meet the test criteria (see text). More details on these polymorphic markers, as well as details for the remaining markers tested (PCR failures and monomorphic microsatellites) are available in Supplementary Table S1. 
$94{ }^{\circ} \mathrm{C}(35 \mathrm{~s}), 58^{\circ} \mathrm{C}(35 \mathrm{~s})$ and $72{ }^{\circ} \mathrm{C}(45 \mathrm{~s})$, then 30 cycles of $94{ }^{\circ} \mathrm{C}(35 \mathrm{~s}), 50{ }^{\circ} \mathrm{C}(35 \mathrm{~s})$ and $72{ }^{\circ} \mathrm{C}(45 \mathrm{~s})$, and finally $10 \mathrm{~min}$ at $72{ }^{\circ} \mathrm{C}$ (ABI 9700 thermal cycler). The amplicons thus generated were diluted 1:30 in water and paired so that two amplicons from the same individual but corresponding to microsatellites with expected PCR bands of different sizes (cf. Table S1) were further processed together. One $\mu \mathrm{L}$ of the plexed diluted amplicon mix was used with $9.5 \mu \mathrm{L}$ of $\mathrm{HiDi}(\mathrm{ABI})$ and $0.5 \mu \mathrm{L}$ of Genescan ROX ladder 50-350 bp (Gel Company) and run on the ABI 3100 Genetic Analyzer. Scoring of fluorescence peaks was performed using GeneMapper4.0 and confirmed manually.

Visual inspection of peak sizes in all test individuals led to the identification of different marker categories (Table 1). Of the 96 test microsatellites, nine had no detectable band in any of the individuals in the panel and 11 had bands in fewer than five of the 11 test individuals. Together, these 20 assays were scored as failed PCR reactions. PCR failures can result from unsuitable primers (e.g. due to sequencing errors in primed region; Long et al. 2007) and/or from the presence of introns in the genomic DNA (either leading to PCR failure or to amplicons of size greater than our maximum detection limit of $350 \mathrm{bp}$ ). Of the remaining primer pairs, eight produced peak patterns that were inconclusive and did not allow accurate genotyping of the test panel. These (8), together with the PCR failures (20), were excluded from the analysis. Twentyeight of the remaining 68 loci were monomorphic in the test panel and 18 of the 40 polymorphic loci had alternative fragments differing in size by a number of nucleotides that was not a multiple of the repeat size. The latter can be insertion-deletion polymorphisms in the microsatellite-containing amplicon and/or non-perfect microsatellites, but remain potentially useful markers. Of the 40 polymorphic loci, 22 were associated with annotated unigenes (Table 2). Ten of the 40 markers might be of limited usefulness: seven showed heterozygote deficiency (commonly found in lepidopterans and usually caused by null alleles; Van't Hof et al. 2007) and three did not meet the criteria for Hardy-Weinberg analysis due to a low-frequency allele in a small sample (Table 2).

We selected 12 of the markers polymorphic in the laboratory population (italics in Table 2 ) to assay in a panel of 24 wild-caught individuals; 12 males and 12 females from South Africa. Three of them (contigs 213, 248, and 3655) had peak patterns that did not allow for clear genotyping and were discarded from further analysis. The remaining nine markers were polymorphic in the wild-caught panel. Three of them were not in HardyWeinberg equilibrium and two pairs were in linkage disequilibrium in the South African population (Table 3), but this was not significant when both test panels were taken into account $(P>0.5$ for all pairwise tests involving the nine markers).

Compared with microsatellites in genomic libraries, those in ESTs generally have lower levels of polymorphism: fewer are polymorphic, they have fewer segregating alleles and the size difference between alleles is smaller (Table 1; Prasad et al. 2005). However, they have important advantages: there are typically fewer problems with PCR amplification when using primers designed

Table 3 EST-based microsatellite markers polymorphic in a wild-caught population

\begin{tabular}{|c|c|c|c|c|c|c|c|c|}
\hline ID & rep size & alleles laboratory & alleles SA & indiv SA & HW SA & $\mathrm{H}_{\mathrm{O}} \mathrm{SA}$ & $\mathrm{H}_{\mathrm{E}} \mathrm{SA}$ & LD SA \\
\hline 455 & 4 & 3 & 3 & 19 & 0.001 & 0.158 & 0.496 & \\
\hline 698 & 2 & 2 & 3 & 18 & 0.013 & 0.111 & 0.294 & \\
\hline 780 & 3 & 3 & 3 & 23 & 0.292 & 0.261 & 0.308 & a \\
\hline$\overline{902}$ & 2 & 2 & 3 & 22 & 1 & 0.409 & 0.394 & \\
\hline 1369 & 4 & 2 & 4 & 18 & 0 & 0.167 & 0.567 & \\
\hline 1710 & 2 & 3 & 2 & 19 & 0.080 & 0.053 & 0.149 & \\
\hline 2555 & 4 & 2 & 3 & 23 & 0.362 & 0.273 & 0.369 & $\mathrm{~b}$ \\
\hline 2904 & 4 & 2 & 3 & 22 & 0.038 & 0.409 & 0.551 & $\mathrm{~b}$ \\
\hline 3410 & 4 & 2 & 3 & 23 & 0.260 & 0.565 & 0.456 & a \\
\hline
\end{tabular}

ID is the contig number with underlining marking a possible 'indel' (i.e. alternative alleles differing by a number of nucleotides that is not a multiple of repeat size); rep size is the number of nucleotides in the microsatellite repeat; alleles laboratory is the number of alleles found in the laboratory panel of 11 butterflies; alleles $S A$ is the number of alleles found in the panel of 24 test butterflies from a wildcaught South African population; indiv $S A$ is the number of individuals from the South African panel that were genotyped for each microsatellite marker; $H W$ is the p-value for the test for deviations from Hardy-Weinberg equilibrium in the South African panel with bold indicating significant deviations; Ho observed frequency of heterozygotes; He expected frequency of heterozygotes; $L D$ corresponds to a letter code for groups of markers in linkage disequilibrium in the South African population: (a) $P=0.0346$, (b) $P=0.0352$, and empty cells having $P>0.16$ for all pairwise LD tests. HW and $L D$ were calculated with GENEPOP (Rousset 2008) based on the genotype data in the Supplementary Table S2. More details on these polymorphic markers, as well as details for the remaining markers tested (including three markers excluded from further analysis) are available in Supplementary Table S2. 
against cDNA sequence and the markers are more likely to be transferable and comparable between species and are valuable in efforts to move from implicated genomic regions to implicated genes. Different recent studies have suggested that ESTs are a good source for microsatellite markers (Wren et al. 2000; Ellis \& Burke 2007). Scanning EST collections for microsatellites can be especially valuable for groups where gDNA-based microsatellite development has proven challenging.

\section{Acknowledgements}

The authors wish to thank Stuart Macdonald and Steven Lockton for help with data collection and analysis, Maaike de Jong and Bas Zwaan for access to biological material from a wild population and Nicolien Pul for technical assistance with DNA preps. This work was supported by funds from the American National Science Foundation (NSF DEB-0235697 to ALD and PB), the Dutch Scientific Research Organization (NWO VENI 863.04.013 to $\mathrm{PB}$ ) and the Portuguese Foundation for Science and Technology (FCT PTDC/BIA-BDE/65295/2006 to PB).

\section{References}

Beldade P, Brakefield PM (2002) The genetics and evo-devo of butterfly wing patterns. Nature Reviews Genetics, 3, 442452.

Beldade P, Rudd S, Gruber JD, Long AD (2006) A wing expressed sequence tag resource for Bicyclus anynana butterflies, an evo-devo model. BMC Genomics, 7, 130.

Beldade P, Mcmillan WO, Papanicolaou A (2008) Butterfly genomics eclosing. Heredity, 100, 150-157.

Beldade P, Saenko SV, Pul N, Long AD (2009) A gene-based linkage map for Bicyclus anynana butterflies allows for a comprehensive analysis of synteny with the lepidopteran reference genome. PLoS Genetics, 5, e1000366.

Brakefield PM, Beldade P, Zwaan BJ (2009) The African butterfly Bicyclus anynana: evolutionary genetics and evo-devo. In: Emerging Model Organisms: A Laboratory Manual, Volume 1: (eds Behringer RR, Johnson AD \& Krumlauf RE) Cold Spring Harbor Laboratory Press, Cold Spring Harbor, NY, 291-330.

Ellegren H (2004) Microsatellites: simple sequences with complex evolution. Nature Reviews. Genetics, 5, 435-445.

Ellis JR, Burke JM (2007) EST-SSRs as a resource for population genetic analyses. Heredity, 99, 125-132.

Fondon JW Iii, Garner HR (2004) Molecular origins of rapid and continuous morphological evolution. Proceedings of the National Academy of Sciences of the United States of America, 101, 1805818063.

Jiggins CD, Mavarez J, Beltran M, Mcmillan WO, Johnston JS, Bermingham E (2005) A genetic linkage map of the mimetic butterfly Heliconius melpomene. Genetics, 171, 557-570.

Kapan DD, Flanagan NS, Tobler A et al. (2006) Localization of Müllerian mimicry genes on a dense linkage map of Heliconius erato. Genetics, 173, 735-757.

Kashi Y, King DG (2006) Simple sequence repeats as advantageous mutators in evolution. Trends in Genetics, 22, 253-259.
Long AD, Beldade P, Macdonald SJ (2007) Estimation of population heterozygosity and library construction-induced mutation rate from expressed sequence tag collections. Genetics, 176, 711-714.

Papanicolaou A, Gebauer-Jung S, Blaxter ML, Owen Mcmillan W, Jiggins CD (2008) ButterflyBase: a platform for lepidopteran genomics. Nucleic Acids Research, 36, D582-D587.

Prasad MD, Muthulakshmi M, Madhu M, Archak S, Mita K, Nagaraju J (2005) Survey and analysis of microsatellites in the silkworm, Bombyx mori: frequency, distribution, mutations, marker potential and their conservation in heterologous species. Genetics, 169, 197-214.

Pringle EG, Baxter SW, Webster CL, Papanicolaou A, Lee SF, Jiggins CD (2007) Synteny and chromosome evolution in the lepidoptera: evidence from mapping in Heliconius melpomene. Genetics, 177, 417-426.

Rockman MV, Wray GA (2002) Abundant raw material for cisregulatory evolution in humans. Molecular Biology and Evolution, 19, 1991-2004.

Rousset F (2008) GENEPOP'007: a complete reimplementation of the GENEPOP software for Windows and Linux. Molecular Ecology Resources 8, 103-106.

Rozen S, Skaletsky HJ (2000) Primer3 on the WWW for general users and for biologist programmers. In: Bioinformatics Methods and Protocols: Methods in Molecular Biology. (eds Krawetz S \& Misener S), pp. 365-386. Humana Press, Totowa, NJ.

Schlötterer C (2004) The evolution of molecular markers - just a matter of fashion? Nature Reviews Genetics, 5, 63-69.

Schuelke M (2000) An economic method for the fluorescent labeling of PCR fragments. Nature Biotechnology, 18, 233234.

Van't Hof AE, Zwaan BJ, Saccheri IJ, Daly D, Bot ANM, Brakefield PM (2005) Characterization of 28 microsatellite loci for the butterfly Bicyclus anynana. Molecular Ecology Notes, 5, 169-172.

Van't Hof AE, Brakefield PM, Saccheri IJ, Zwaan BJ (2007) Evolutionary dynamics of multilocus microsatellite arrangements in the genome of the butterfly Bicyclus anynana, with implications for other Lepidoptera. Heredity, 98, 320-328.

Van't Hof AE, Marec F, Saccheri IJ, Brakefield PM, Zwaan BJ (2008) Cytogenetic characterization and AFLP-based genetic linkage mapping for the butterfly Bicyclus anynana, covering all 28 karyotyped chromosomes. PLoS ONE, 3, e3882.

Wang B, Porter AH (2004) An AFLP-based interspecific linkage map of sympatric, hybridizing Colias butterflies. Genetics, 168, 215-225.

Wren JD, Forgacs E, Fondon JW Iii et al. (2000) Repeat polymorphisms within gene regions: phenotypic and evolutionary implications. American Journal of Human Genetics, 67, 345-356.

Yasukochi Y, Ashakumary LA, Baba K, Yoshido A, Sahara K (2006) A second generation integrated map of the silkworm reveals synteny and conserved gene order between lepidopteran insects. Genetics, 173, 1319-1328.

Zhang DX (2004) Lepidopteran microsatellite DNA: redundant but promising. Trends in Ecology \& Evolution, 19, 507-509.

\section{Supporting Information}

Additional supporting information may be found in the online version of this article. 
Table S1 Details for all 96 microsatellites tested in the laboratory panel

Table S2 Details for the 12 microsatellites tested in the wildcaught panel

Please note: Wiley-Blackwell are not responsible for the content or functionality of any supporting information supplied by the authors. Any queries (other than missing material) should be directed to the corresponding author for the article.

doi: 10.1111/j.1755-0998.2009.02691.x

C 2009 Blackwell Publishing Ltd

\title{
Characterization of microsatellite loci isolated from the wasp, Microstigmus nigrophthalmus (Hymenoptera)
}

\author{
ERIC R. LUCAS, ${ }^{*}$ GAVIN J. HORSBURGH, ${ }^{*}$ DEBORAH A. DAWSON* and JEREMY FIELD十 \\ *NERC Molecular Genetics Facility, Department of Animal and Plant Sciences, University of Sheffield, Sheffield S10 2TN, UK, \\ +Department of Biology and Environmental Science, University of Sussex, Brighton BN1 9QG, UK
}

\begin{abstract}
Fifty-two microsatellite loci were characterized in 22-31 unrelated females of the wasp (Microstigmus nigrophthalmus) collected from the Mata do Paraiso, Viçosa, M.G., Brazil. Fifty-one of these loci were developed from a microsatellite-enriched genomic library derived from $M$. nigrophthalmus and one was derived from the wasp, Ormyrus nitidulus. The genus Microstigmus represents an independent origin of social behaviour in the Hymenoptera and is thus of great potential in the study of social evolution.
\end{abstract}

Keywords: Hymenoptera, microsatellite, Microstigmus nigrophthalmus, social organization, wasp

Received 21 December 2008; revision accepted 24 March 2009

Microstigmus is a neotropical genus of apoid wasp characterized by the construction of nests using a combination of external organic material and silk produced by adult females. In contrast to most other apoid wasps, Microstigmus demonstrates evidence of social organization (Matthews 1968, 1991). The species Microstigmus nigrophthalmus (Melo 1992) lives in groups of one to five females (Melo \& Campos 1993) and has great potential for use in the study of social evolution. We describe the identification of a set of polymorphic dinucleotide microsatellite loci for $M$. nigrophthalmus.

Genomic DNA was extracted from the thorax and abdomen of a single female $M$. nigrophthalmus pupa (046P1) captured from the Mata do Paraiso, Viçosa, M.G., Brazil. Genomic DNA was digested with $\mathrm{MboI}$ and sizeselected (250-750 bp). The restriction fragments were enriched for $(\mathrm{CA})_{\mathrm{n}}$ and $(\mathrm{GA})_{\mathrm{n}}$ and their complements, as described by Armour et al. (1994) but without the preenrichment hybridization PCR-amplification step. Enriched fragments were ligated into BamHI-digested, CIP-dephosphorylated pBluescript SK+ (Stratagene) and screened for $(\mathrm{CA})_{\mathrm{n}}$ and $(\mathrm{GA})_{\mathrm{n}}$ and their complements.

Correspondence: Jeremy Field, Fax: +44 (0) 1273 678937;

E-mail: j.field@sussex.ac.uk
Positive clones were sequenced in both directions, a consensus sequence created and primers designed using Primer 3 (Rozen \& Skaletsky 2000). One-hundred and seven unique sequences were identified using BLASTN v.2.2.4 (Altschul et al. 1997, EMBL accession numbers FM957351-FM957457). Primer sets were designed for 86 loci with at least seven tandem repeat units.

As a result of the haplodiploid sex determination system found in the Hymenoptera, no part of the nuclear genome exists exclusively in one sex. Adult males and females of this species can be distinguished based on morphology. In addition, males are haploid, whereas females are diploid. All individuals genotyped for the characterization of our loci were female. Thirty-seven females were captured from the Mata do Paraiso (see above) and stored in $1 \mathrm{~mL}$ of absolute ethanol in screw-capped microfuge tubes at room temperature for several months, then in a $-20{ }^{\circ} \mathrm{C}$ freezer. Genomic DNA was extracted from whole thoraces and each locus tested for amplification and polymorphism with between 22 and 31 unrelated individuals from the Mata do Paraiso population. Each $10-\mu \mathrm{L}$ PCR contained approximately $50 \mathrm{ng}$ of genomic DNA, $1.0 \mu \mathrm{M}$ of each primer, $0.20 \mathrm{~mm}$ of each dNTP, $2.0 \mathrm{~mm} \mathrm{MgCl}_{2}$ and 0.25 units of Taq DNA polymerase (Biotaq; Bioline) in the manufacturer's buffer. PCR 